\title{
Correction to: No evidence for a role of trills in male response to territorial intrusion in a complex singer, the Thrush Nightingale
}

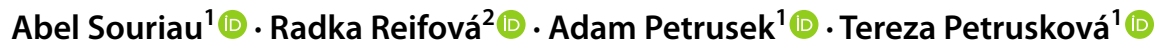

Published online: 30 April 2021

(c) Deutsche Ornithologen-Gesellschaft e.V. 2021

Correction to: Journal of Ornithology

https://doi.org/10.1007/s10336-021-01879-9

In the original publication of the article, "Availability of data and materials" section was published with an error.

It is given as "Sound recordings used to generate spectrograms (Fig. 1, Fig. 2, Online resource 1) are provided at https://doi.org/10.7479/b478-jj11" but it should be "All experimental recordings used in the analysis have been deposited in the Animal Sound Archive at the Museum for Natural History in Berlin and are available under the CCBY-SA Licence at the following https://doi.org/10.7479/ b478-jj11".
The original article has been updated.

Publisher's Note Springer Nature remains neutral with regard to jurisdictional claims in published maps and institutional affiliations.

The original article can be found online at https://doi.org/10.1007/ s10336-021-01879-9.

Abel Souriau

abel.souriau@gmail.com

1 Department of Ecology, Faculty of Science, Charles University, Viničná 7, 12844 Praha 2, Prague, Czech Republic

2 Department of Zoology, Faculty of Science, Charles University, Prague, Czech Republic 